\title{
Lithium limits trimethyltin-induced cytotoxicity and proinflammatory response in microglia without affecting the concurrent autophagy impairment
}

\author{
Cinzia Fabrizi ${ }^{\mathbf{a}}$, Elena Pompilia*, Francesca Somma ${ }^{\mathbf{a}}$, Stefania De Vito ${ }^{\mathbf{a}}$, \\ Viviana Ciraci ${ }^{\mathbf{a}}$, Marco Artico ${ }^{\mathbf{b}}$, Paola Lenzic ${ }^{\mathbf{c}}$, Francesco Fornai ${ }^{\mathbf{c}, \mathbf{d}}$ \\ and Lorenzo Fumagallia
}

\begin{abstract}
Trimethyltin (TMT) is a highly toxic molecule present as an environmental contaminant causing neurodegeneration particularly of the limbic system both in humans and in rodents. We recently described the occurrence of impairment in the late stages of autophagy in TMT-intoxicated astrocytes. Here we show that similarly to astrocytes also in microglia, TMT induces the precocious block of autophagy indicated by the accumulation of the autophagosome marker, microtubule associated protein light chain 3. Consistent with autophagy impairment we observe in TMT-treated microglia the accumulation of p62/SQSTM1, a protein specifically degraded through this pathway. Lithium has been proved effective in limiting neurodegenerations and, in particular, in ameliorating symptoms of TMT intoxication in rodents. In our in vitro model, lithium displays a pro-survival and anti-inflammatory action reducing both cell death and the proinflammatory response of TMT-treated microglia. In particular, lithium exerts these activities without reducing TMT-induced accumulation of light chain 3 protein. In fact, the autophagic block imposed by TMT is unaffected by lithium administration. These results are of interest as defects in the execution of autophagy are frequently observed in neurodegenerative diseases and lithium is considered a promising therapeutic agent for these pathologies. Thus, it is relevant that this cation can still maintain its pro-survival and anti-inflammatory role in conditions of autophagy block. Copyright $\odot 2016$ John Wiley \& Sons, Ltd.
\end{abstract}

Keywords: microglia; trimethyltin; autophagy; neurodegeneration; neuroinflammation

\section{Introduction}

Trimethyltin (TMT) is a highly neurotoxic molecule belonging to the family of organotins. These compounds during the last decades have been used for a variety of industrial and agricultural applications as pesticides, fungicides and antifouling paints for ship hulls (Appel, 2004). Some of them have been banned such as tributyltin chloride in the United States during the 1980s (Fent, 1996) but despite their toxicity methyltins are still heavily used as heat stabilizers in polyvinyl chloride production (Dobson et al., 2005). Leaching of organotins from polyvinyl chloride material has been proposed as one of the principal sources of environmental contamination (Richardson \& Edwards, 2009) and in fact, they can be detected as contaminants in fresh water, tap water, seawater, algae and fish (Borghi \& Porte, 2002; Fent, 1996; Mundy \& Freudenrich, 2006). Although methyltin is more widely used, dimethyltin is characterized by low direct toxicity and its conversion into TMT by the addition of a methyl group has been reported

Q2 to occur in vivo (Furuhashi et al., 2008).

TMT damages specific populations of neurons and in particular the limbic system, including the pyriform/enthorinal cortex, olfactory bulb, amygdala and hippocampal formation (Chang \& Dyer, 1985; Kawada et al., 2008). The main symptoms of TMT intoxication include aggressive behavior, disorientation, seizures and severe memory loss (Kreyberg et al., 1992; Reuhl \& Cranmer, 1984). Similarly to other neurodegenerative disorders, TMT-induced neurodegeneration is characterized by neuronal selectivity and neuroinflammation is always accompanied by both astroglial and microglial activation (Geloso et al., 2011; Pompili et al., 2004, Q3 2011). In the hippocampus of TMT-intoxicated rodents, microglia activation is observed during the first post-intoxication day and then persists over a long period (Koczyk \& Oderfeld-Nowak, 2000). At the same time in the hippocampus, proinflammatory cytokines are upregulated (Bruccoleri \& Harry, 2000). TMT administered in vitro to microglial cultures determines the increase in the expression of M1 markers (tumor necrosis factor [TNF]- $\alpha$, interleukin [IL]-1 $\beta$, IL-6 and inducible nitric oxide synthase) and the decrease of the M2 markers (CD206 and arginase-1) (Kim et al., 2014). Recently, TMT was demonstrated to activate directly the BV-2

*Correspondence to: Elena Pompili, Department of Anatomy, Histology, Forensic Medicine and Orthopedics, Sapienza University, Via Alfonso Borelli, 50, 00161 Rome, Italy. E-mail: elena.pompili@uniroma1.it

${ }^{a}$ Department of Anatomy, Histology, Forensic Medicine and Orthopedics, Sapienza University, Rome, Italy

${ }^{b}$ Department of Sensory Organs, Sapienza University, Rome, Italy

'Department of Human Morphology and Applied Biology, University of Pisa, Pisa, Italy

dIRCCS Neuromed, Pozzilli, Italy 
microglial cell line via the NADPH oxidase-dependent reactive oxygen species generation (Kim \& Kim, 2015).

A general response of cells exposed to stressful conditions is the activation of autophagy. This evolutionary conserved catabolic pathway aims to remove damaged organelles and misfolded proteins and generally displays a cytoprotective function (Nikoletopoulou et al., 2013). It is initiated by the formation of a double-membrane vesicle known as an autophagosome, which engulfs aggregated proteins or entire organelles. After fusion with lysosomes, the cargo is finally degraded (for a recent review see Klionsky et al., 2016). Our previous data showed the importance of the autophagic pathway in improving the survival of neuronal cells exposed to TMT (Fabrizi et al., 2012, 2014). Moreover, we recently reported that in astrocytes, TMT determines the impairment of the late stages of autophagy limiting autophagosome movement and thus their maturation into autophagolysosomes (Fabrizi et al., 2016).

In this paper, we wanted to answer the question as to whether the TMT-induced impairment of autophagy was restricted to astrocytes or instead could be a possible common feature of the reaction of glial cells to this toxic compound. Thus, we extended our previous observations to microglia, which are considered the resident macrophages of the central nervous system. During neurodegenerative diseases, microglial cells become activated and can produce highly toxic molecules such as radical species leading to further neuronal dysfunction and neurodegeneration.

Moreover, as lithium neuroprotective activity has been proved in many different experimental settings, including TMT intoxication (Kim et al., 2013; Yoneyama et al., 2014), we studied if lithium could limit microglial response to TMT in our culture model system. Interestingly, lithium has been shown to modulate the autophagic pathway functioning both as an inducer of autophagy at low concentrations $(0.5 \mathrm{~mm})$ and as an inhibitor of the same pathway when used at concentrations higher than $2 \mathrm{~mm}$ (Sarkar \& Rubinsztein, 2006; Sarkar et al., 2008). Owing to its multiple molecular targets, the lithium mechanism of action is still elusive (for a review, see Chiu et al., 2013).

\section{Materials and methods}

\section{Cell cultures and treatments}

All procedures were carried out in accordance with Italian laws and guidelines established for the care and use of animals in research. Microglial cells were prepared from the cortex of newborn (P4) Sprague-Dawley rats, as described previously (Pompili et al., 2011). Briefly, after 14 days in culture, microglial cells were separated from the underlying astrocytic monolayer by gentle agitation and cells were cultured in Dulbecco minimal Eagle's medium

Q4 (Invitrogen, Jtaly) supplemented with $10 \%$ fetal calf serum (Sigma Milan, Italy) in $5 \% \mathrm{CO}_{2}$. Cultures routinely consisted of $\geq 95 \%$ microglial cells as determined by staining with Griffonia simplicifolia isolectin B4 (Sigma, St. Louis, AO, USA).

Microglial cells $\left(1 \times 10^{5}\right.$ cells cm $\left.{ }^{-2}\right)$ were seeded on to $96-, 24-$ or six-well plates depending on the experiment. After $24 \mathrm{~h}$ from seeding cells were treated with TMT $(10 \mu \mathrm{m}$; Heraeus, Karlsrube, Germany), lithium chloride $(0.5,1,2 \mathrm{~mm}), 0.5 \mu \mathrm{M}$ rapamycin, $10 \mu \mathrm{M}$ indirubin-3'-monoxime, $20 \mathrm{~nm}$ SB-216763, $100 \mathrm{~nm}$ bafilomycin A1 and $10 \mathrm{~mm} 3$-methyladenine. All these compounds were used alone or in combination for different times ranging from 3 to $48 \mathrm{~h}$ as reported in the figure legends. All reagents were from Q5 Sigma-Aldrich unless otherwise stated.

\section{Assessment of cell viability}

Cell death was evaluated by measuring the release of lactate dehydrogenase in culture medium by the Cytotoxicity Detection Kit (Roche, Mannheim, Germany) according to the manufacturer's protocols.

\section{Terminal deoxynucleotidyl transferase-mediated biotinylated UTP nick end labeling}

The DNA fragmentation of apoptotic cells was detected using the terminal deoxynucleotidyl transferase-mediated biotinylated UTP nick end labeling (TUNEL) kit (In Situ Cell Death Detection Kit; Roche). Cells were cultured on coverslips and at the end of the drug treatment fixed in $4 \%$ paraformaldehyde in $0.1 \mathrm{M}$ phosphate buffer pH 7.4 (PBS) at room temperature for $15 \mathrm{~min}$ and then incubated with a permeabilizing solution ( $0.1 \%$ Triton $X-100)$ for $10 \mathrm{~min}$ at $4^{\circ} \mathrm{C}$. The cells were then incubated with the TUNEL reaction mixture for $60 \mathrm{~min}$ at $37^{\circ} \mathrm{C}$ and visualized by inverted fluorescence microscopy (Eclipse E600; Nikon Instruments SpA, Firenze, Italy). Q6 TUNEL-positive nuclei were counted in 10 non-overlapping fields per coverslip and then converted to percentage by comparing TUNEL-positive counts with the total cell nuclei visualized by 4',6'-diamino-2-phenylindole counterstaining.

\section{Monodansylcadaverine}

Living cells plated on coverslips were incubated with $0.05 \mathrm{~mm}$ monodansylcadaverine (MDC; Sigma) (Biederbick et al., 1995) in Q7 PBS at $37^{\circ} \mathrm{C}$ for $10 \mathrm{~min}$. Cells were washed three times in PBS and then immediately analyzed by fluorescence microscopy.

\section{Western blotting}

Treated and untreated cells were lysed in RIPA buffer containing protease and phosphatase inhibitors (Protease Inhibitor Cocktail and Phosphatase Inhibitor Cocktail 2; Sigma). To detect both solu- Q8 ble and insoluble aggregates of p62/SQSTM1, cells were lysed directly in $100 \mu \mathrm{l} 2 \times$ sodium dodecyl sulfate (SDS)-polyacrylamide gel electrophoresis gel loading buffer (125 mm Tris- $-\mathrm{HCl}$, pH 7.4, $4 \%$ SDS, $0.04 \%$ bromophenol blue, $30 \mathrm{mg} \mathrm{ml}^{-1}$ DTT added immediately before use), sonicated three times for $5 \mathrm{~s}$ and then boiled for 5 min (Bjørkøy et al., 2009). All samples were clarified by centrifugation at $1000 \mathrm{rpm}_{\lambda}$ for $5 \mathrm{~min}$. Equivalent amount of protein Q9 $(10 \mu \mathrm{g})$ from each sample was electrophoretically resolved on $12.5 \%$ precast SDS-polyacrylamide gels (ExcelGel; GE Healthcare Biosciences) using horizontal apparatus (Pharmacia Biotech, Upp- Q10 sala, Sweden). Then, separated proteins were electro-transferred on to nitrocellulose membranes (Schleicher \& Schuell) by a semi- Q11 dry system (Novablot; Pharmacia Biotech). Membranes were blocked with $3 \%$ non-fat milk in PBS and then incubated (overnight at $4{ }^{\circ} \mathrm{C}$ ) with the following antibodies: anti-light chain (LC) 3B (Sigma; 1: 1000 in 3\% bovine serum albumin in PBS), anti- Q12 p62/SQSTM1 (MBL, PM045; 1: 1000 in 3\% non-fat milk in PBS). After extensive washing with PBS containing $0.1 \%$ Tween-20, blots were incubated with 1: 2000 dilution of horseradish peroxidaseconjugated secondary antibody (Amersham Bioseiences) for $1 \mathrm{~h}$ Q13 at room temperature. Immunopositive bands were detected with a chemiluminescence detection system (GE Healthcare Biosciences). To check for equal loading of the gel, membranes were stripped and reprobed with mouse anti- $\beta$-actin or anti-GAPDH 
Q14 antibodies (1: 20 000; Sigma). Densitometric analysis was perQ15formed with the Quantity One software (Bio-Rad Laboratories).

\section{Enzyme-linked immunosorbent assay}

The release of TNF- $\alpha$ and IL-10 into culture supernatants was determined by standard enzyme-linked immunosorbent assay techniques according to the manufacturer's instructions (R\&D Q16 Systems, MN, USA).

\section{Animals and immunohistochemistry}

Male Sprague-Dawley rats (7 weeks old, weighing $250 \mathrm{~g}$ ) (Charles River, Italy) were housed in a temperature- and humiditycontrolled room $\left(21 \pm 5^{\circ} \mathrm{C}\right.$ and $60 \%$ humidity) and fed ad libitum with standard laboratory diet and water. All procedures were carried out in accordance with Italian laws and guidelines established for the care and the use of animals in research. Rats received a single intraperitoneal dose of TMT $\left(8 \mathrm{mg} \mathrm{kg}^{-1}\right.$ body weight; Heraeus) or vehicle (saline) and were killed at 7 days after treatment. Brains were rapidly removed, embedded in OCT com-

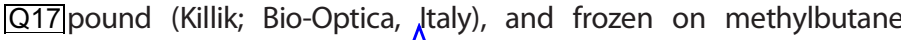
precooled with liquid nitrogen as previously reported (Pompili et al., 2011).

Sagittal cryostatic sections ( $7 \mu \mathrm{m}$ thickness) from saline and TMT-treated rats were fixed with $4 \%$ paraformaldehyde in PBS ( $\mathrm{pH} 7.4)$ at room temperature for $10 \mathrm{~min}$. After quenching autofluorescence with $0.05 \mathrm{M}$ ammonium chloride and saturation of non-specific sites with 3\% normal donkey serum (BioCell Research Laboratories, Rancho Dominguez, CA, USA) and 0.1\% Triton X-100, sections were incubated overnight at $4{ }^{\circ} \mathrm{C}$ with rabbit anti-p62/ SQSTM1 (1: 500; MBL, PM045) and mouse anti-OX42 (1: 100; Serotec, Oxford, UK). After washing, the sections were incubated with a mixture of donkey Dy-light 549 antirabbit IgG (1: 400; Jackson ImmunoResearch Laboratories, West Grove, PA, USA) and donkey Dy-light 488-labeled antimouse IgG (1: 200; Jackson ImmunoResearch Laboratories). Negative controls were performed substituting specific immunoglobulins with an equivalent amount of non-specific immunoglobulins and omitting primary antibodies. Slides were mounted with Vectashield mounting medium, containing 4',6'-diamino-2-phenylindole for nuclear staining (Vector Laboratories, Burlingame, CA, USA). Examinations and photographs were made using a fluorescence microscope (Eclipse E600; Nikon Instruments SpA).

\section{Statistics}

Statistical analyses were conducted using GraphPad Prism version 4.00 software. Data are expressed as averages \pm SD. Comparisons were analyzed using one-way ANOVA with the Bonferronicorrected $t$-test. All experiments were performed in triple and reproduced at least three times.

\section{Results}

In the experiments presented below we used the minimum con- Q18 centration of TMT $(10 \mu \mathrm{M})$ previously demonstrated to affect microglial cell viability (Pompili et al., 2011). Consistently with those previously published data, at 24 and $48 \mathrm{~h}$ of TMT treatment a progressive increment respective to control in lactate dehydro- Q19 genase release (indicative of necrosis and late apoptosis) was measured in the culture medium of rat microglial primary cultures (Fig. 1A). At earlier time points, a statistically significant increase F1 with respect to the untreated control in the percentage of TUNEL-positive apoptotic cells could be measured at $8 \mathrm{~h}$ of TMT treatment, which then increased further at $24 \mathrm{~h}$ (Fig. 1B).

As we already demonstrated the appearance of autophagic vesicles in both neuronal and astrocyte cultures exposed to TMT (Fabrizi et al., 2012, 2016), we then checked for the presence of these vesicles also in TMT-treated microglia. To that end, microglial cells were first labeled by the fluorescent dye MDC, a compound that marks autophagic vesicles (Vázquez \& Colombo, 2009). As shown in Fig. 2, TMT determined the appearance of many MDC- F2 positive structures that were not observed if this compound was administered in combination with the autophagic inhibitor 3-methyladenine known to block autophagy at the sequestration stage (Stroikin et al., 2004). MDC-positive vesicles were also detected, as expected, after treatment with the autophagic inducer rapamycin. In addition to late-stage autophagosomes, MDC was also reported to label acidic endosomes and lysosomes (Munafo \& Colombo, 2001). Thus, to confirm the increased presence of autophagosomes in TMT-treated microglia, we measured on Western blot the amount of the lipidated form of light chain 3 (LC3-II) (Fig. 3). In fact, LC3-II binds tightly to the autophagosomal

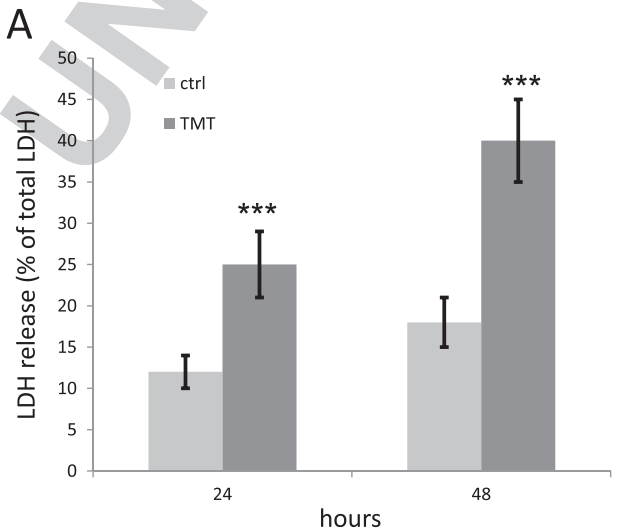

B

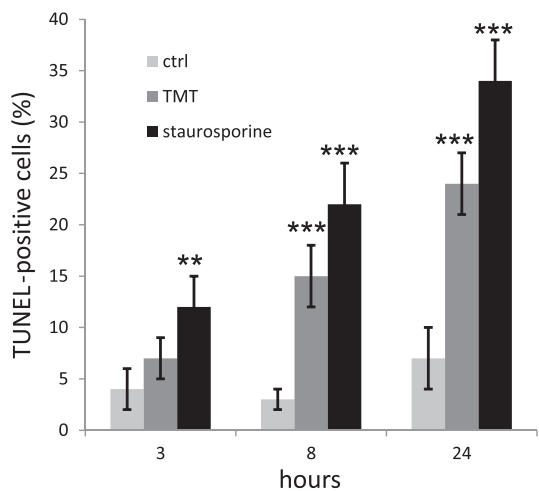

Figure 1. TMT cytotoxicity in microglial primary cultures. (A) LDH release in the culture medium of microglia treated with TMT for 24 and $48 \mathrm{~h}$. (B) Apoptotic cells labeled by TUNEL in microglial cultures treated with TMT or staurosporine $(0.5 \mu \mathrm{m}$; positive control) at 3, 8 and $24 \mathrm{~h}$. Each data point represents the average $\pm \mathrm{SD}$ of four independent experiments. ANOVA with Bonferroni's corrected $t$-test. ${ }^{* *} P<0.01,{ }^{* * *} P<0.001$ versus untreated control. Ctrl, control; LDH, lactic dehydrogenase; TMT, trimethyltin; TUNEL, terminal deoxynucleotidyl transferase-mediated biotinylated UTP nick end labeling. 

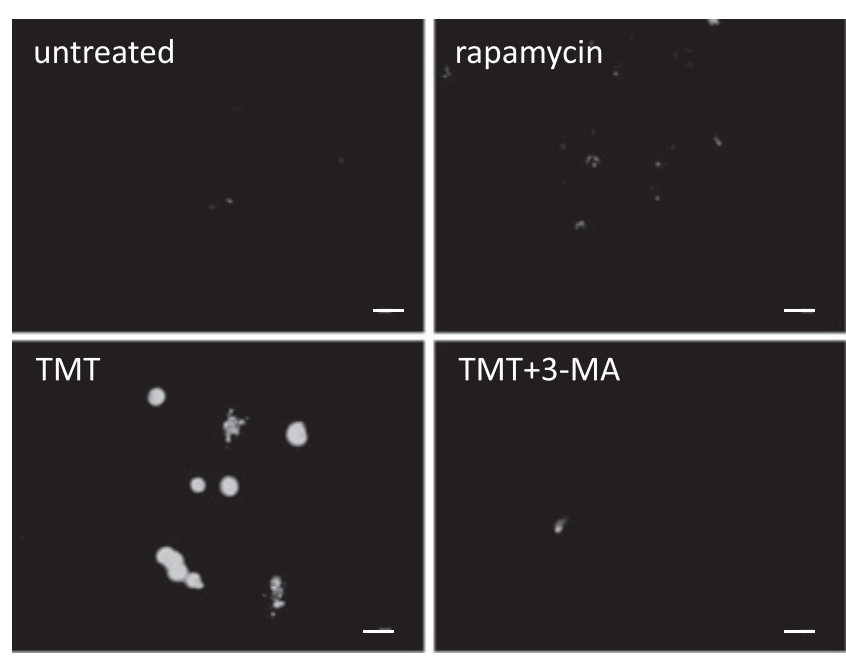

Figure 2. Autophagic vesicles detected by monodansylcadaverine staining. Microglial cells were left untreated or treated for $24 \mathrm{~h}$ with TMT alone, TMT-positive $10 \mathrm{~mm}$ 3-MA or $0.5 \mu \mathrm{m}$ rapamycin. Monodansylcadaverinepositive vesicles were detected in TMT-treated samples. These vesicles were not observed if TMT was added in combination with the autophagy inhibitor 3-MA. Results obtained using the autophagy inducer rapamycin are shown as positive control. Scale bar $=50 \mu \mathrm{m}$. 3-MA, 3-methyladenine; TMT, trimethyltin.
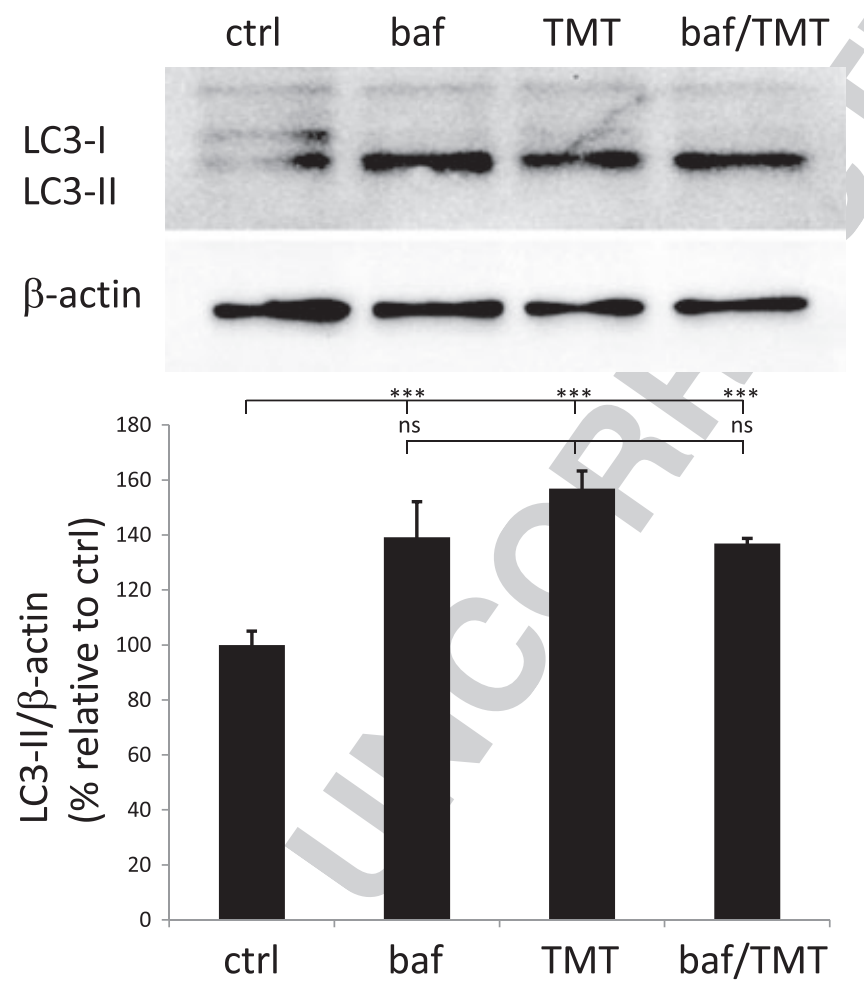

Figure 3. LC3 turnover assay in TMT-treated microglia in the presence of a lysosomal inhibitor. (A) Western blot analysis of LC3-I/II conversion in microglia treated for $3 \mathrm{~h}$ with TMT alone or in combination with $100 \mathrm{~mm}$ baf; (B) Densitometric analysis of western blot data shown in (A). Each data point represents the average $\pm S D$ of three independent experiments. ANOVA with Bonferroni's corrected $t$-test. ${ }^{* * *} P<0.001$ versus untreated control; baf, bafilomycin A1; ctrl, control; LC3, light chain 3; ns, not significant; TMT, trimethyltin.

membrane and is considered a reliable autophagosome marker (Kabeya et al., 2000). When treated with TMT the amount of
LC3-II increased rapidly in microglia with respect to the untreated control (Fig. 3). This result does not necessarily indicate an increased autophagic flux as it could be also related to an impairment in the maturation of autophagic vesicles (Klionsky et al., 2016). Thus, we analyzed LC3-II levels in microglia in the presence of bafilomycin A1. This compound is a potent inhibitor of the vacuolar-type $\mathrm{H}^{+}$ATPase, which impairs lysosomal functions blocking late stages of autophagy and consequently LC3 degradation (Boya et al., 2005; Yamamoto et al., 1998). When TMT and bafilomycin $A 1$ were administered in combination no further increase in LC3-II/ $\beta$-actin ratio was measured indicating that TMT was already blocking the autophagosome maturation and consequent LC3-II degradation (Fig. 3). The observed impairment in autophagy induced by TMT persisted in up to $48 \mathrm{~h}$ of treatment (not shown).

As mentioned above, lithium is a modulator of autophagy known to limit cell death and inflammatory response in many different experimental models (Chiu et al., 2013).

When added to microglia this cation was able to reduce significantly the TMT toxicity when used at 1-2 mM (Fig. 4). Lithium F4 cytoprotective action against TMT was also confirmed by the MTT test (not shown). Concentrations of lithium above $1 \mathrm{~mm}$ are known to inhibit the glycogen kinase synthase-3 (GSK-3) (Sarkar et al., 2008). Other GSK-3 inhibitors, such as indirubin-3'-monoxime or SB-216763 similarly to lithium limited TMT toxicity in microglia (not shown).

In addition to its cytoprotective action, lithium was also able to reduce the TMT-induced release of TNF- $\alpha$. At the same time this cation favored the production of the anti-inflammatory cytokine, IL-10 (Fig. 5).

Lithium is also known to affect the autophagic pathway. Thus, we decided to check if lithium pro-survival and anti-inflammatory actions observed in our model could be related to its ability to

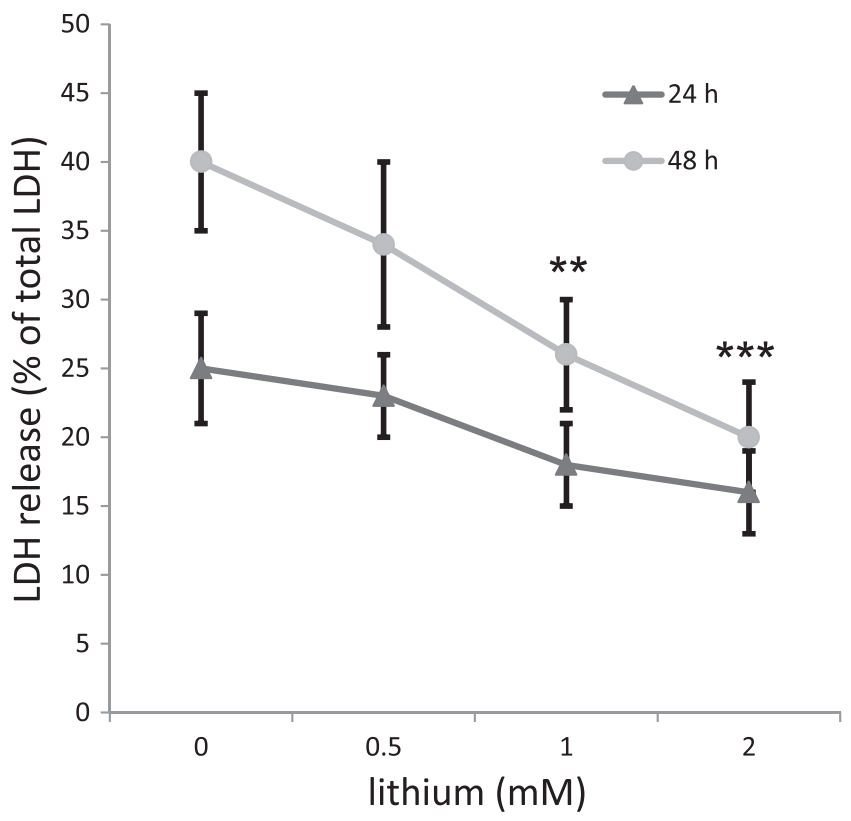

Figure 4. Lithium protects microglial cells from trimethyltin toxicity. Microglial cells were treated with trimethyltin alone or in combination with lithium (0-2 mM) for 24 or $48 \mathrm{~h}$. Cell viability was assessed by LDH release. Averages \pm SD of four independent experiments. ANOVA with Bonferroni's corrected $t$-test. ${ }^{* *} P<0.01,{ }^{* *} P<0.001$ versus untreated control. LDH, lactic dehydrogenase. 
A

A

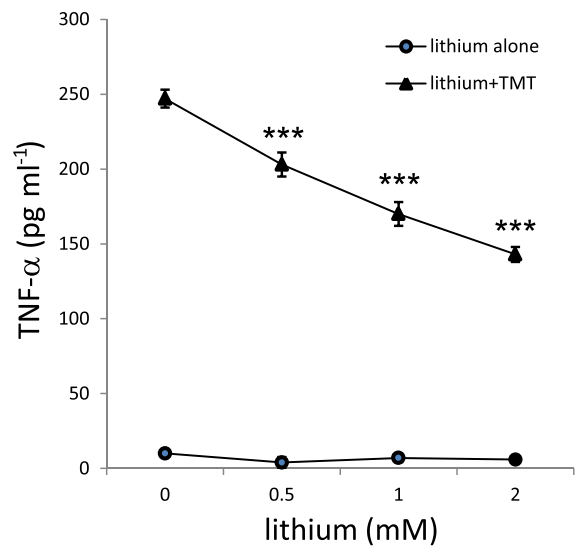

B

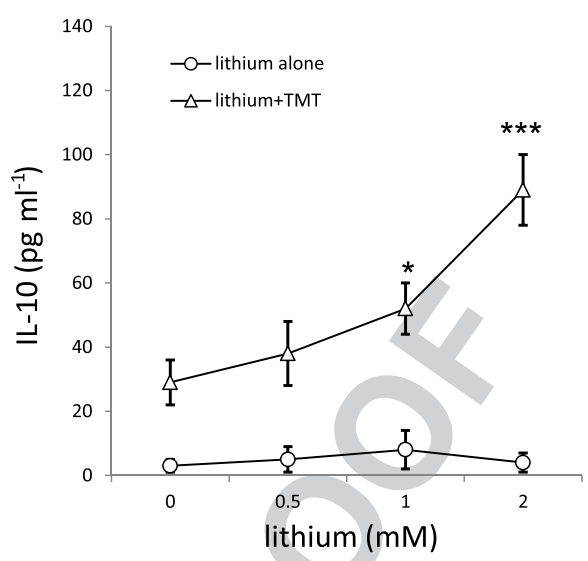

Figure 5. Immunomodulatory action of lithium in TMT-treated microglia. Microglial cells were treated for $16 \mathrm{~h}$ with TMT alone or in combination with lithium $(0.5,1,2 \mathrm{~mm})$ and the levels of TNF- $\alpha$ and IL-10 in the supernatants measured by enzyme-linked immunosorbent assay. Averages \pm SD of three independent experiments. ANOVA with Bonferroni's corrected $t$-test. ${ }^{*} P<0.05$, ${ }^{* *} P<0.001$ versus TMT alone. IL, interleukin; TMT, trimethyltin; TNF, tumor necrosis factor.

force the autophagic impairment induced by TMT. The analysis of LC3-I/II conversion and p62 (also known as SQTSM1) expression revealed that lithium was not able to revert the autophagic block imposed by TMT at none of the concentrations used ranging from

F6 0.5 to $2 \mathrm{~mm}$ (Fig. $6 \mathrm{~A}, \mathrm{~B}$ ). In particular, this is the same range of lithium concentrations shown before to protect microglia from TMT toxicity and to limit its proinflammatory response (Figs 4 and 5). Figure 6 also shows the levels of expression of LC3 and p62/SQSTM1 measured in TMT-treated astrocytes that were higher with respect to the ones obtained in microglia using the same Q21 experimental settings.

Moreover, when we checked for the expression of p62/SQSTM1 in brain slices of intoxicated rats most of the immunoreactivity was observed in astrocytes as previously reported (Fabrizi et al., 2016),

F7 while only few OX-42-positive microglial cells were stained (Fig. 7). In control sections obtained from rats treated with saline only, p62/SQSTM1 was hardly detectable (Fig. 7).

\section{Discussion}

Our observations of TMT-treated microglial cultures showed the appearance of many MDC-positive vesicles and an increased LC3-I/II conversion after exposure to this toxic compound. Both these results are indicative of autophagosome formation. Besides, the analysis of the autophagic flux during TMT treatment indicated a block of autophagy at its late stages as no further increase in the LC3-II/ $\beta$-actin ratio was observed after the combined treatment of this organotin with bafilomycin A1 (lysosomal inhibitor). The accumulation of p62/SQSTM1 in TMT-treated samples is in line with the impairment in autophagy as this protein is preferentially degraded through this catabolic route (Bjørkøy et al., 2005). We recently described the occurrence of an autophagic block in astrocytes intoxicated with TMT (Fabrizi et al., 2016). Although an autophagic block was observed in both astrocytes and microglia following TMT treatment, this compound appeared to induce a much higher accumulation of LC3-II and p62/SQSTM1 in the former population of glial cells with respect to the latter. Similarly the expression of p62/SQSTM1 in the brain of intoxicated animals was mostly associated to astrocytes (Fabrizi et al., 2016) while less evident in microglia (present data). Although in vitro and in vivo data are in general

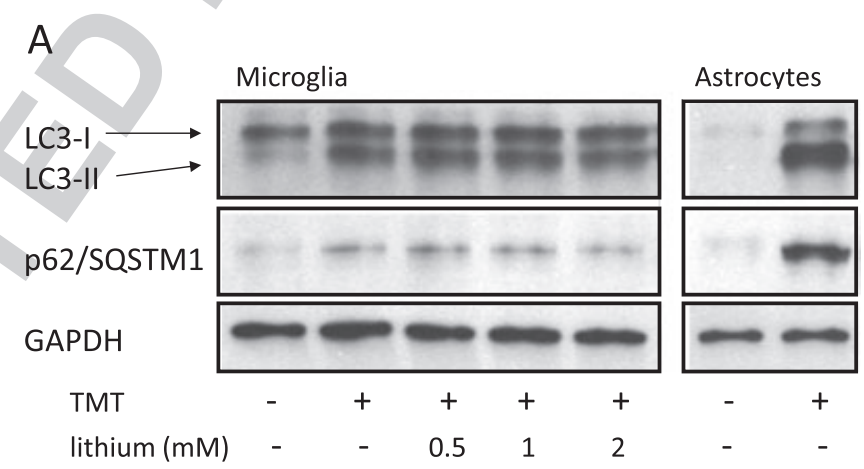

B

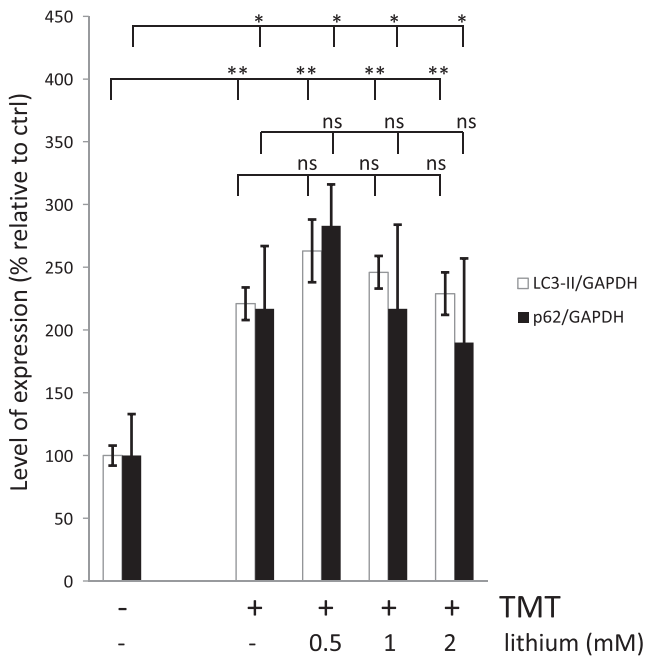

Figure 6. Lithium is ineffective in modifying LC3 and p62/SQSTM1 accumulation in TMT-treated microglia. (A) Western blot analysis of LC3-I/II conversion and p62/SQSTM1 expression in primary microglia treated for $24 \mathrm{~h}$ with TMT alone or in combination with lithium $(0.5,1$ and $2 \mathrm{mM})$. GAPDH is shown as the loading reference control. Level of expression of LC3-1/II and $\mathrm{p62/SQSTM} 1$ in astrocytes treated for $24 \mathrm{~h}$ with TMT is shown for comparison. (B) Densitometric analysis of Western blot data shown in (A). Averages $\pm \mathrm{SD}$ of three independent experiments. ANOVA with Bonferroni's corrected $t$-test. ${ }^{*} P<0.05,{ }^{* *} P<0.01$ versus untreated control; ns, not significant versus TMT alone. Ctrl, control; LC3, light chain 3; TMT, trimethyltin. 


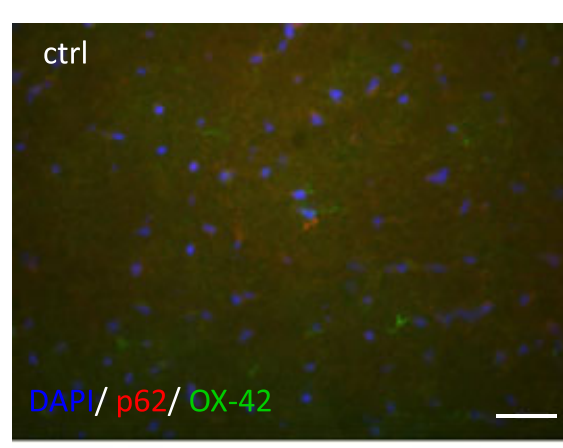

7-day TMT
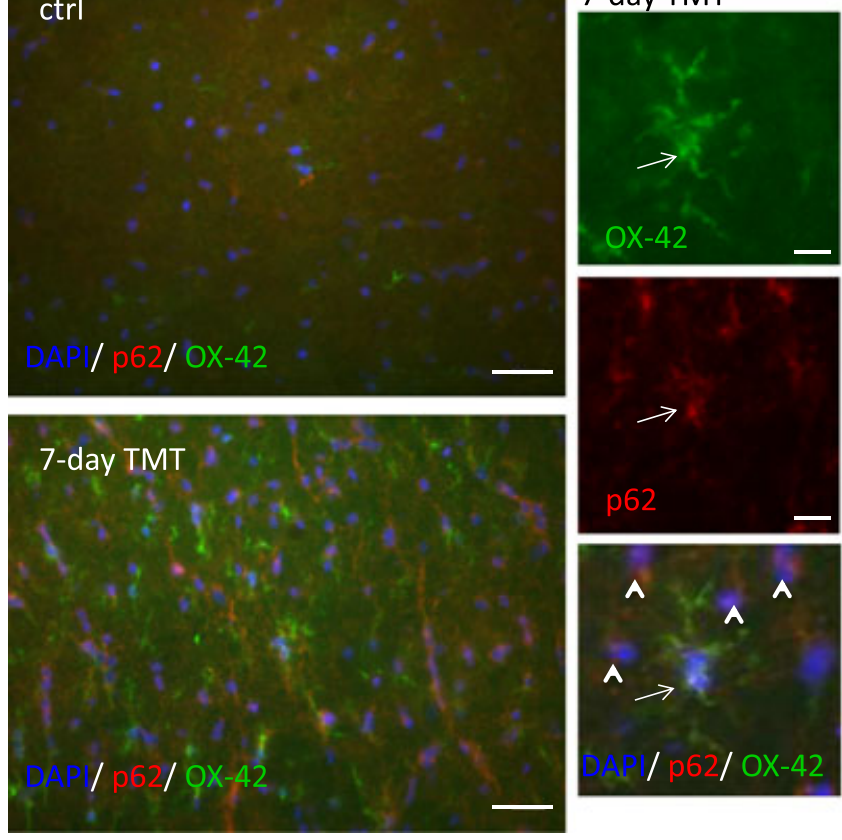

Figure 7. Detection of p62/SQSTM1 in brain slices of TMT intoxicated rats. Immunofluorescence analysis of OX-42 and p62/SQSTM1 in the CA3/hilus hippocampal subfields from rats killed 7 days after TMT intoxication or treated with saline only (ctrl). Nuclei are counterstained with DAPI. Scale bar $=20 \mu \mathrm{m}$. Magnification of an OX-42-positive microglial cell present in TMT-intoxicated brain is shown on the right to visualize better the p62/ SQSTM1 localization; the p62/SQSTM1 staining is also present in many OX-42 cells (arrows). Scale bar $=6 \mu \mathrm{m}$. ctrl, control; DAPI, 4',6'-diamino-2phenylindole; TMT, trimethyltin.

difficult to compare, they are both indicative of a higher tendency of astrocytes to accumulate autophagosomes with respect to microglia during an autophagic block, which could be related to their high cytoplasmic/nuclear ratio.

The observed occurrence of an autophagic block during TMT intoxication in glial cells could be possibly due to the engulfment of autophagic vesicles with undigested materials, which cells are unable to process further. In fact, only some microorganisms such as bacteria (such as Pseudomonas) and phytoplankton have the ability to degrade organotin compounds under certain conditions (Hoch, 2001; Sampath et al., 2012). In particular, inhibition of the autophagic flux and the p62/SQSTM1-dependent activation of the Keap1-Nrf2 pathway has been reported to occur following exposure to other toxic molecules, such as arsenic (Lau et al., 2013). Besides, the Nrf2/p62 signaling pathway is involved in apoptosis resistance from cadmium exposure (Son et al., 2014).

During the past few decades, an increased number of accidental poisonings in workers acutely exposed to methyltins was reported due to their wide industrial use (Tang et al., 2008). Currently, no effective treatment is available for these patients.

Recently, in rodents, lithium was shown to ameliorate the symptoms of TMT intoxication. In fact, this cation can rescue seizures and ameliorate memory deficits in TMT-intoxicated rodents, increasing at the same time as the inhibitory phosphorylation of

Q22 GSK-3 in the hippocampus (Kim et al., 2013). Moreover, lithium enhances neuritogenesis ameliorating the depression-like behavior observed in mice treated with TMT (Yoneyama et al., 2014).

During neurodegenerative diseases, neuronal loss is always accompanied by activation of the glia and in TMT-intoxicated brains activated microglial cells are present in affected brain areas such as the hippocampus (Koczyk \& Oderfeld-Nowak, 2000). In these regions, the expression of proinflammatory cytokines results upregulated (Bruccoleri \& Harry, 2000), and chronic and exaggerated inflammatory responses by microglia can be potentially detrimental for neurons further damaging brain tissue. Thus, wide-ranging anti-inflammatory agents with different blood-brain barrier permeability are currently used in clinical trials for different neurodegenerative diseases to suppress proinflammatory cytokine production by chronically activated microglia (for a recent review see Perry \& Holmes, 2014).

Our data are in line with previous reports showing a beneficial effect of lithium administration in TMT-intoxicated rodents (Kim et al., 2013; Yoneyama et al., 2014). In fact, we show that lithium at least in vitro limits the release of a proinflammatory mediator such as TNF- $\alpha$ potentiating at the same time the production of the anti-inflammatory cytokine, IL-10.

The mechanism of action of lithium is often difficult to assess for its multiple molecular targets, which include, among others, inositol monophosphatase and some related phosphomonoesterases, phosphoglucomutase and GSK-3 (O'Brien \& Klein, 2009). In particular, lithium can inhibit GSK-3 both indirectly and directly, competing with magnesium at its catalytic site; the Ki for GSK-3 inhibition at typical intracellular magnesium concentrations is approximately $1.0 \mathrm{~mm}$ (Ryves \& Harwood, 2001). GSK-3 is known to promote the inflammatory response (Martin et al., 2005) and lithium, such as other GSK-3 inhibitors, has been demonstrated to limit the production of inflammatory mediators in macrophages (Park et al., 2011), dendritic cells (Rodionova et al., 2007) and microglia (Huang et al., 2009; Yuskaitis \& Jope, 2009).

Interestingly, in our microglial cultures, lithium could display its pro-survival and immunomodulatory action without being able to overcome the autophagic block imposed by TMT.

This appears particularly relevant because this cation, alone or in combination with valproic acid, is considered a promising agent for treating neurodegenerative diseases (Chiu et al., 2013) and common features of these pathologies are protein misfolding, aggregation and deposition, which are indicative of the possible failure of catabolic processes in general and of autophagy in particular (Vidal et al., 2014).

In conclusion, our data indicate that lithium could potentially maintain its beneficial effects when used for the treatment of pathologic conditions, such as some neurodegenerative diseases, during which an autophagic impairment is observed.

\section{Acknowledgements}

These studies were supported by grants awarded by Università Sapienza (Ricerche Universitarie) to LF.

\section{Conflict of interest}

The authors did not report any conflict of interest.

\section{References}

Appel KE. 2004. Organotin compounds: toxicokinetic aspects. Drug Metab. Rev. 36(3-4): 763-786.

Biederbick A, Kern HF, Elsässer HP. 1995. Monodansylcadaverine (MDC) is a specific in vivo marker for autophagic vacuoles. Eur. J. Cell Biol. 66: 3-14.

Bjørkøy G, Lamark T, Brech A, Outzen H, Perander M, Overvatn A, Stenmark $H$, Johansen T. 2005. p62/SQSTM1 forms protein aggregates degraded by autophagy and has a protective effect on huntingtin-induced cell death. J. Cell Biol. 171(4): 603-614. 
Bjørkøy G, Lamark T, Pankiv S, Øvervatn A, Brech A, Johansen T. 2009. Monitoring autophagic degradation of p62/SQSTM1. Methods Enzymol. 452: 181-197.

Borghi V, Porte C. 2002. Organotin pollution in deep-sea fish from the northwestern Mediterranean. Environ. Sci. Technol. 36(20): 4224-4228.

Boya P, Gonzalez-Polo RA, Casares N, Perfettini JL, Dessen P, Larochette N, Metivier D, Meley D, Souquere S, Yoshimori T et al. 2005. Inhibition of macroautophagy triggers apoptosis. Mol. Cell. Biol. 25: 1025-1040.

Bruccoleri A, Harry GJ. 2000. Chemical-induced hippocampal neurodegeneration and elevations in TNFalpha, TNFbeta, IL-1alpha, IP-10, and MCP1 mRNA in osteopetrotic (op/op) mice. J. Neurosci. Res. 62: 146-155.

Chang LW, Dyer RS. 1985. Septotemporal gradients of trimethyltin-induced hippocampal lesions. Neurobehav. Toxicol. Teratol. 7: 43-49.

Chiu CT, Wang Z, Hunsberger JG, Chuang DM. 2013. Therapeutic potential of mood stabilizers lithium and valproic acid: beyond bipolar disorder. Pharmacol. Rev. 65: 105-142.

Dobson S, Howe PD, Floyd P. 2005. Concise international chemical assessment document. International Programme on Chemical Safety; 2005. Mono- and disubstituted methyltin, butyltin, and octyltin compounds. http://www.inchem.org/documents/cicads/cicads/cicad73.pdf (accessed September 9, 2014).

Fabrizi C, Somma F, Pompili E, Biagioni F, Lenzi P, Fornai F, Fumagalli L. 2012. Role of autophagy inhibitors and inducers in modulating the toxicity of trimethyltin in neuronal cell cultures. J. Neural Transm. 119(11): 1295-1305.

Fabrizi C, De Vito S, Somma F, Pompili E, Catizone A, Leone S, Lenzi P, Fornai F, Fumagalli L. 2014. Lithium improves survival of PC12 pheochromocytoma cells in high-density cultures and after exposure to toxic compounds. Int. J. Cell Biol. 2014: 135908.

Fabrizi C, Pompili E, De Vito S, Somma F, Catizone A, Ricci G, Lenzi P, Fornai F, Fumagalli L. 2016. Impairment of the autophagic flux in astrocytes intoxicated by trimethyltin. Neurotoxicology 52: 12-22.

Fent K. 1996. Ecotoxicology of organotin compounds. Crit. Rev. Toxicol. 26 $1-117$.

Furuhashi K, Ogawa M, Suzuki Y, Endo Y, Kim Y, Ichihara G. 2008. Methylation of dimethyltin in mice and rats. Chem. Res. Toxicol. 21(2): 467-471.

Geloso MC, Corvino V, Michetti F. 2011. Trimethyltin-induced hippocampal degeneration as a tool to investigate neurodegenerative processes. Neurochem. Int. 58(7): 729-738.

Hoch M. 2001. Organotin compounds in the environment-an overview. Appl. Geochem. 16: 719-743.

Huang WC, Lin YS, Wang CY, Tsai CC, Tseng HC, Chen CL, Lu PJ, Chen PS, Qian L, Hong JS, Lin CF. 2009. Glycogen synthase kinase-3 negatively regulates anti-inflammatory interleukin-10 for lipopolysaccharideinduced iNOS/NO biosynthesis and RANTES production in microglial cells. Immunology 128(1 Suppl.): e275-e286.

Kabeya Y, Mizushima N, Ueno T, Yamamoto A, Kirisako T, Noda T, Kominami E, Ohsumi Y, Yoshimori T. 2000. LC3, a mammalian homologue of yeast Apg8p, is localized in autophagosome membranes after processing. EMBO J. 19(21): 5720-5728.

Kawada K, Yoneyama M, Nagashima R, Ogita K. 2008. In vivo acute treatment with trimethyltin chloride causes neuronal degeneration in the murine olfactory bulb and anterior olfactory nucleus by different cascades in each region. J. Neurosci. Res. 86(7): 1635-1646.

da Kim J, Kim YS. 2015. Trimethyltin-induced microglial activation via NADPH oxidase and MAPKs pathway in BV-2 microglial cells. Mediat. Inflamm. 2015: 729509.

Kim J, Yang M, Kim SH, Kim JC, Wang H, Shin T, Moon C. 2013. Possible role of the glycogen synthase kinase-3 signaling pathway in trimethyltininduced hippocampal neurodegeneration in mice. PLoS One 8(8) e70356.

Kim J, Yang M, Son Y, Jang H, Kim D, Kim JC, Kim SH, Kang MJ, Im HI, Shin T, Moon C. 2014. Glial activation with concurrent up-regulation of inflammatory mediators in trimethyltin-induced neurotoxicity in mice. Acta Histochem. 116(8): 1490-1500.

Klionsky DJ, Abdelmohsen K, Abe A, Abedin MJ, Abeliovich $\mathrm{H}$, Acevedo Arozena A, Adachi H, Adams CM, Adams PD et al. 2016. Guidelines for the use and interpretation of assays for monitoring autophagy (3rd edition). Autophagy 12: 1-222.

Koczyk D, Oderfeld-Nowak B. 2000. Long-term microglial and astroglial activation in the hippocampus of trimethyltin-intoxicated rat: stimulation of NGF and TrkA immunoreactivities in astroglia but not in microglia. Int. J. Dev. Neurosci. 18(6): 591-606.

Kreyberg S, Torvik A, Bjørneboe A, Wiik-Larsen W, Jacobsen D. 1992. Trimethyltin poisoning: report of a case with postmortem examination. Clin. Neuropathol. 11(5): 256-259.
Lau A, Zheng Y, Tao S, Wang H, Whitman SA, White E, Zhang DD. 2013. Arsenic inhibits autophagic flux, activating the Nrf2-Keap1 pathway in a p62-dependent manner. Mol. Cell. Biol. 33(12): 2436-2446.

Martin M, Rehani K, Jope RS, Michalek SM. 2005. Toll-like receptor-mediated cytokine production is differentially regulated by glycogen synthase kinase 3. Nat. Immunol. 6(8): 777-784.

Munafo DB, Colombo MI. 2001. A novel assay to study autophagy: Regulation of autophagosome vacuole size by amino acid deprivation. J. Cell Sci. 114: 3619-3629.

Mundy WR, Freudenrich TM. 2006. Apoptosis of cerebellar granule cells induced by organotin compounds found in drinking water: involvement of MAP kinases. Neurotoxicology 27: 71-81.

Nikoletopoulou V, Markaki M, Palikaras K, Tavernarakis N. 2013. Crosstalk between apoptosis, necrosis and autophagy. Biochim. Biophys. Acta 1833(12): 3448-3459.

O'Brien WT, Klein PS. 2009. Validating GSK3 as an in vivo target of lithium action. Biochem. Soc. Trans. 37(Pt 5): 1133-1138.

Park SH, Park-Min KH, Chen J, Hu X, Ivashkiv LB. 2011. Tumor necrosis factor induces GSK3 kinase-mediated cross-tolerance to endotoxin in macrophages. Nat. Immunol. 12(7): 607-615.

Perry VH, Holmes C. 2014. Microglial priming in neurodegenerative disease. Nat. Rev. Neurol. 10(4): 217-224.

Pompili E, Nori SL, Geloso MC, Guadagni E, Corvino V, Michetti F, Fumagalli L. 2004. Trimethyltin-induced differential expression of PAR subtypes in reactive astrocytes of the rat hippocampus. Brain Res. Mol. Brain Res. 122: 93-98.

Pompili E, Fabrizi C, Nori SL, Panetta B, Geloso MC, Corvino V, Michetti F, Fumagalli L. 2011. Protease-activated receptor-1 expression in rat microglia after trimethyltin treatment. J. Histochem. Cytochem. 59(3): 302-311.

Reuhl KR, Cranmer JM. 1984. Developmental neuropathology of organotin compounds. Neurotoxicology 5(2): 187-204.

Richardson R, Edwards M. 2009. Vinyl chloride and organotin stabilizers in water contacting new and aged PVC pipes. Water Research Foundation. http://www.waterrf.org/PublicReportLibrary/2991.pdf.

Rodionova E, Conzelmann M, Maraskovsky E, Hess M, Kirsch M, Giese T, Ho AD, Zöller M, Dreger P, Luft T. 2007. GSK-3 mediates differentiation and activation of proinflammatory dendritic cells. Blood 109(4): 1584-1592.

Ryves WJ, Harwood AJ. 2001. Lithium inhibits glycogen synthase kinase-3 by competition for magnesium. Biochem. Biophys. Res. Commun. 280 (3): 720-725.

Sampath R, Venkatakrishnan H, Ravichandran V, Chaudhury RR. 2012. Biochemistry of TBT-degrading marine pseudomonads isolated from Indian coastal waters. Water Air Soil Pollut. 223: 99-106.

Sarkar S, Rubinsztein DC. 2006. Inositol and IP3 levels regulate autophagy: biology and therapeutic speculations. Autophagy 2(2): 132-134.

Sarkar S, Krishna G, Imarisio S, Saiki S, O'Kane CJ, Rubinsztein DC. 2008. A rational mechanism for combination treatment of Huntington's disease using lithium and rapamycin. Hum. Mol. Genet. 17(2): 170-178.

Son YO, Pratheeshkumar P, Roy RV, Hitron JA, Wang L, Zhang Z, Shi X. 2014. $\mathrm{Nrf2/p62} \mathrm{signaling} \mathrm{in} \mathrm{apoptosis} \mathrm{resistance} \mathrm{and} \mathrm{its} \mathrm{role} \mathrm{in} \mathrm{cadmium-}$ induced carcinogenesis. J. Biol. Chem. 289(41): 28660-28675.

Stroikin Y, Dalen H, Lööf S, Terman A. 2004. Inhibition of autophagy with 3methyladenine results in impaired turnover of lysosomes and accumulation of lipofuscin-like material. Eur. J. Cell Biol. 83: 583-590.

Tang X, Xia L, Chen J, Huang M, Lai G, Li S, Wu B, Wang J, Zhu G. 2008. Clinical analysis on 76 cases from 13 poisoning accidents caused by trimethyltin chloride. Chin. Occup. Med. 35: 91-94.

Vázquez CL, Colombo Ml. 2009. Assays to assess autophagy induction and fusion of autophagic vacuoles with a degradative compartment, using monodansylcadaverine (MDC) and DQ-BSA. Methods Enzymol. 452: 85-95.

Vidal RL, Matus S, Bargsted L, Hetz C. 2014. Targeting autophagy in neurodegenerative diseases. Trends Pharmacol. Sci. 35(11): 583-591.

Yamamoto A, Tagawa Y, Yoshimori T, Moriyama Y, Masaki R, Tashiro Y. 1998. Bafilomycin A1 prevents maturation of autophagic vacuoles by inhibiting fusion between autophagosomes and lysosomes in rat hepatoma cell line, H-4-II-E cells. Cell Struct. Funct. 23: 33-42.

Yoneyama M, Shiba T, Hasebe S, Umeda K, Yamaguchi T, Ogita K. 2014. Lithium promotes neuronal repair and ameliorates depression-like behavior following trimethyltin-induced neuronal loss in the dentate gyrus. PLoS One $\mathbf{9}(2)$ e87953.

Yuskaitis CJ, Jope RS. 2009. Glycogen synthase kinase-3 regulates microglial migration, inflammation, and inflammation-induced neurotoxicity. Cell. Signal. 21(2): 264-273. 


\section{Author Query Form}

\section{Journal: Journal of Applied Toxicology}

\section{Article: jat_3344}

Dear Author,

During the copyediting of your paper, the following queries arose. Please respond to these by annotating your proofs with the necessary changes/additions.

- If you intend to annotate your proof electronically, please refer to the E-annotation guidelines.

- If you intend to annotate your proof by means of hard-copy mark-up, please use the standard proofing marks. If manually writing corrections on your proof and returning it by fax, do not write too close to the edge of the paper. Please remember that illegible mark-ups may delay publication.

Whether you opt for hard-copy or electronic annotation of your proofs, we recommend that you provide additional clarification of answers to queries by entering your answers on the query sheet, in addition to the text mark-up.

\begin{tabular}{|c|c|c|}
\hline Query No. & Query & Remark \\
\hline Q1 & $\begin{array}{l}\text { AUTHOR: Please confirm that given names (red) and surnames/family names ( green) } \\
\text { have been identified correctly. }\end{array}$ & \\
\hline Q2 & $\begin{array}{l}\text { AUTHOR: Although methyltin is more widely used, dimethyltin is characterized by low } \\
\text { direct toxicity and its conversion into TMT by the addition of a methyl group has been } \\
\text { reported to occur in vivo-OK now? }\end{array}$ & \\
\hline Q3 & $\begin{array}{l}\text { AUTHOR: Similarly to other neurodegenerative disorders, TMT-induced } \\
\text { neurodegeneration is characterized by neuronal selectivity and neuroinflammation is } \\
\text { always accompanied by both astroglial and microglial activation-OK now? }\end{array}$ & \\
\hline Q4 & AUTHOR: Invitrogen, Italy_-where in Italy? & $\overline{\overline{\overline{\sqrt{ }}}}$ \\
\hline Q5 & $\begin{array}{l}\text { AUTHOR: Sigma-Aldrich-pls add address (other } 2 x \text { Sigma companies are Sigma alone } \\
\text { and from Italy and the USA)? }\end{array}$ & $\bar{\equiv}$ \\
\hline Q6 & AUTHOR: Nikon Instruments SpA, Firenze, Italy-OK now? & $\overline{\overline{\bar{\tau}}}$ \\
\hline Q7 & AUTHOR: Sigma—which Sigma office is this? & $\equiv$ \\
\hline Q8 & AUTHOR: Sigma—which Sigma office is this? & 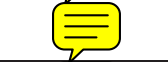 \\
\hline Q9 & AUTHOR: centrifugation at $1000 \mathrm{rpm}$ for $5 \mathrm{~min}$ — pls add units in ' $\mathrm{g}$ ' not rpm & $\bar{\equiv}$ \\
\hline Q10 & AUTHOR: GE Healthcare Biosciences—pls add address & $\equiv$ \\
\hline Q11 & AUTHOR: Schleicher \& Schuell—pls add address & $\overline{\overline{\bar{\nu}}}$ \\
\hline Q12 & AUTHOR: Sigma—which Sigma office is this? & $\bar{\equiv}$ \\
\hline Q13 & AUTHOR: Amersham Biosciences_-pls add address & $\sqrt{\overline{\overline{2}}}$ \\
\hline Q14 & AUTHOR: Sigma—which Sigma office is this? & $\overline{\overline{\bar{v}}}$ \\
\hline Q15 & AUTHOR: Bio-Rad Laboratories—pls add address & $\overline{\bar{\nu}}$ \\
\hline Q16 & AUTHOR: R\&D Systems, MN, USA—where in MN, USA? & $\overline{\overline{\bar{V}}}$ \\
\hline Q17 & AUTHOR: Bio-Optica, Italy_—where in Italy? & $\overline{\bar{\nu}}$ \\
\hline Q18 & $\begin{array}{l}\text { AUTHOR: In the experiments presented below-Is 'below' correct here (as Materials \& } \\
\text { methods is above this section)? }\end{array}$ & $\bar{\equiv}$ \\
\hline
\end{tabular}




\begin{tabular}{|c|l|c|} 
Query No. & \multicolumn{1}{|c|}{ Query } & Remark \\
\hline Q19 & $\begin{array}{l}\text { AUTHOR: Consistently with those previously published data, at 24 and 48h of TMT } \\
\text { treatment a progressive increment respective to control—OK now? }\end{array}$ \\
\hline Q20 & AUTHOR: Vázquez \& Colombo, 2009-OK now (have changed to match therefs list)? \\
\hline Q21 & $\begin{array}{l}\text { AUTHOR: Figure 6 also shows the levels of expression of LC3 and p62/SQSTM1 } \\
\text { measured in TMT-treated astrocytes that were higher with respect to the ones obtained } \\
\text { in microglia using the same experimental settings.-OK now? }\end{array}$ & $\begin{array}{l}\text { AUTHOR: In fact, this cation can rescue seizures and ameliorate memory deficits in } \\
\text { TMT-intoxicated rodents, increasing at the same time as the inhibitory phosphorylation } \\
\text { of GSK-3 in the hippocampus-OK now? }\end{array}$ \\
\hline Q23 & AUTHOR: The authors did not report any conflict of interest.-OK now? \\
\hline Q24 & AUTHOR: Please provide the page range for Reference Yoneyama et al., 2014 & \\
\hline
\end{tabular}


Required software to e-Annotate PDFs: Adobe Acrobat Professional or Adobe Reader (version 7.0 or above). (Note that this document uses screenshots from Adobe Reader $\mathrm{X}$ )

The latest version of Acrobat Reader can be downloaded for free at: http://get.adobe.com/uk/reader/

Once you have Acrobat Reader open on your computer, click on the Comment tab at the right of the toolbar:

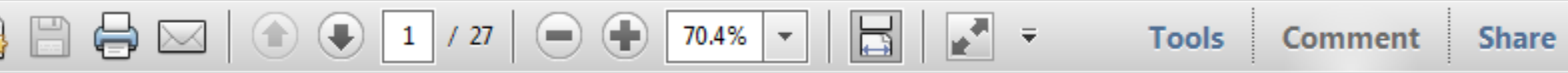

This will open up a panel down the right side of the document. The majority of tools you will use for annotating your proof will be in the Annotations section, pictured opposite. We've picked out some of these tools below:

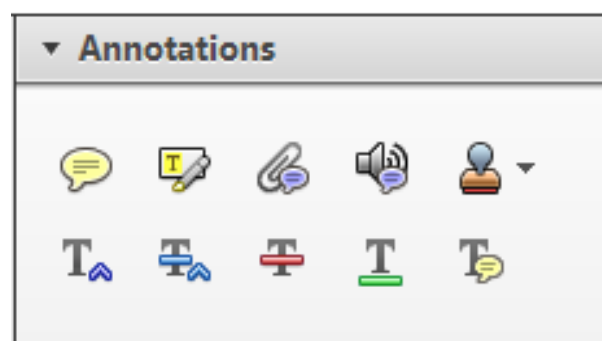

1. Replace (Ins) Tool - for replacing text.

Strikes a line through text and opens up a text box where replacement text can be entered.

How to use it

- Highlight a word or sentence.

- Click on the Replace (Ins) icon in the Annotations section.

- Type the replacement text into the blue box that appears.

Idard tramework for the analysis of $\mathrm{m}$ icy-Nevertheless, it also led to exog،

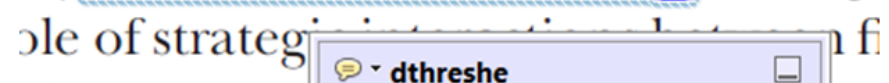
aber of comp 08/06/2011 15:58:17 is that the s1 nain compo: be level, are exc nc

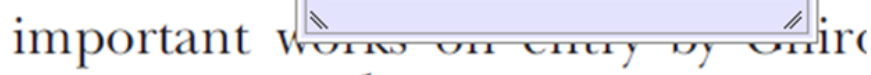
M heneferth) ${ }^{1}$ we anen the "hlarl $\mathrm{l}$

3. Add note to text Tool - for highlighting a section to be changed to bold or italic.

T Highlights text in yellow and opens up a text box where comments can be entered.

\section{How to use it}

- Highlight the relevant section of text.

- Click on the Add note to text icon in the Annotations section.

- Type instruction on what should be changed regarding the text into the yellow box that appears.

namic responses of mark ups ent with the VAR evidence

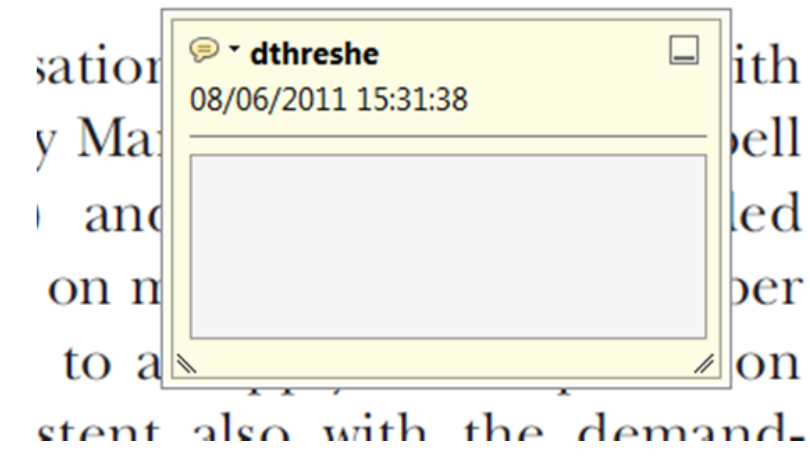

2. Strikethrough (Del) Tool - for deleting text.

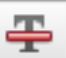

Strikes a red line through text that is to be deleted.

How to use it

- Highlight a word or sentence.

- Click on the Strikethrough (Del) icon in the Annotations section.

there is no room tor extra prohts al c ups are zero and the number of ret) values are not determined by Blanchard and Kiyotaki (1987), sfect competition in general equilil ts of aggregate demand and supply lassical framework assuming monol eph on evorenous number of firme

4. Add sticky note Tool - for making notes at specific points in the text.

Marks a point in the proof where a comment needs to be highlighted.

How to use it

- Click on the Add sticky note icon in the Annotations section.

- Click at the point in the proof where the comment should be inserted.

- Type the comment into the yellow box that appears.

iaisu airu suppiy sirucks. hivsl ui

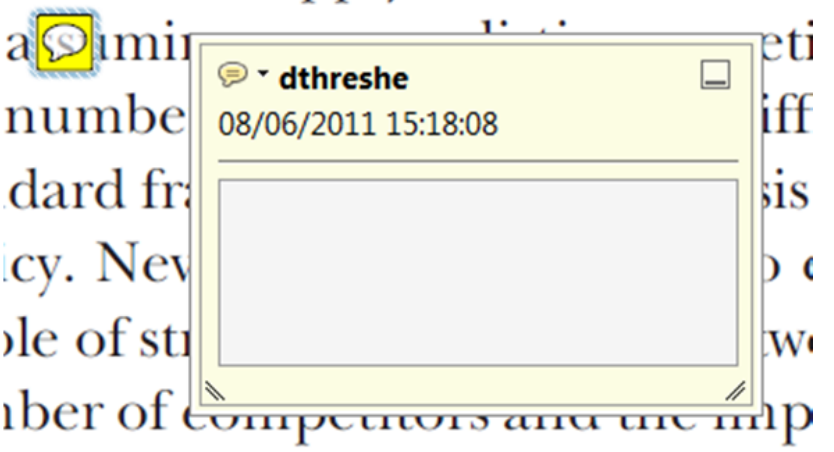

is that the structure of the secto. 
5. Attach File Tool - for inserting large amounts of text or replacement figures.

Inserts an icon linking to the attached file in the appropriate pace in the text.

How to use it

- Click on the Attach File icon in the Annotations section.

- Click on the proof to where you'd like the attached file to be linked.

- Select the file to be attached from your computer or network.

- Select the colour and type of icon that will appear in the proof. Click OK.

E N D

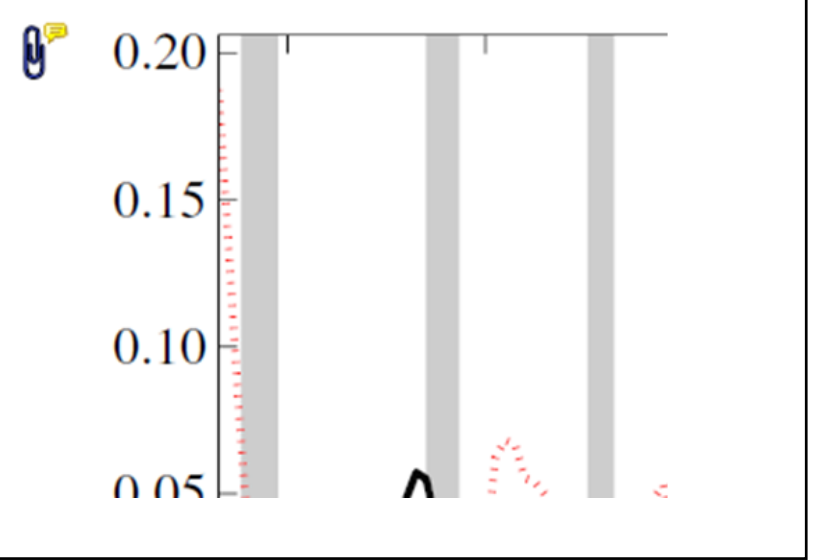

6. Add stamp Tool - for approving a proof if no corrections are required.

- Inserts a selected stamp onto an appropriate place in the proof.

\section{How to use it}

- Click on the Add stamp icon in the Annotations section.

- $\quad$ Select the stamp you want to use. (The Approved stamp is usually available directly in the menu that appears).

- Click on the proof where you'd like the stamp to appear. (Where a proof is to be approved as it is, this would normally be on the first page).

or the business cycie, starting with the on perfect competition, constant ret

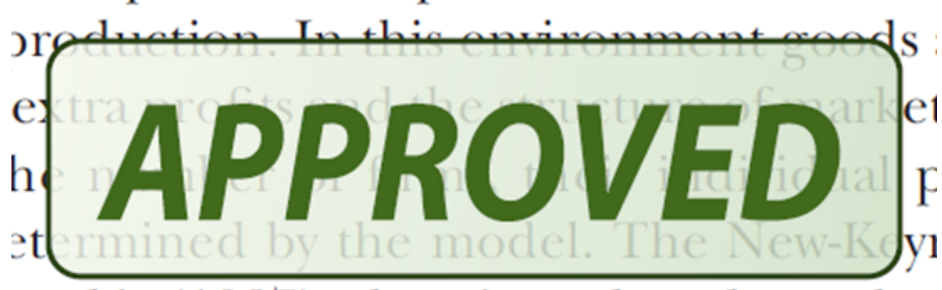
otaki (1987), has introduced produc general equilibrium models with nomin:

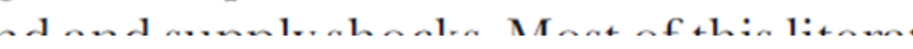

- Drawing Markups

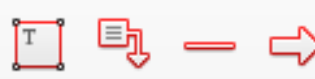

$0 \bigcirc \sqrt{6} \otimes$

\section{How to use it}

- Click on one of the shapes in the Drawing Markups section.

- Click on the proof at the relevant point and draw the selected shape with the cursor.

- To add a comment to the drawn shape, move the cursor over the shape until an arrowhead appears.

- Double click on the shape and type any text in the red box that appears.
7. Drawing Markups Tools - for drawing shapes, lines and freeform annotations on proofs and commenting on these marks.

Allows shapes, lines and freeform annotations to be drawn on proofs and for comment to be made on these marks.

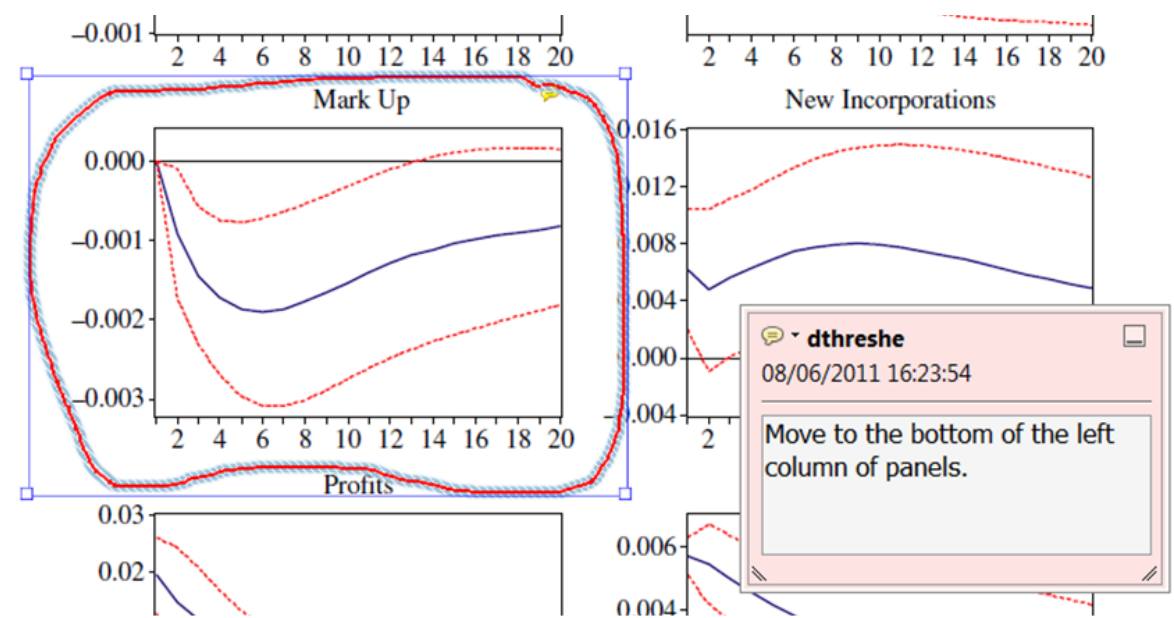

For further information on how to annotate proofs, click on the Help menu to reveal a list of further options:

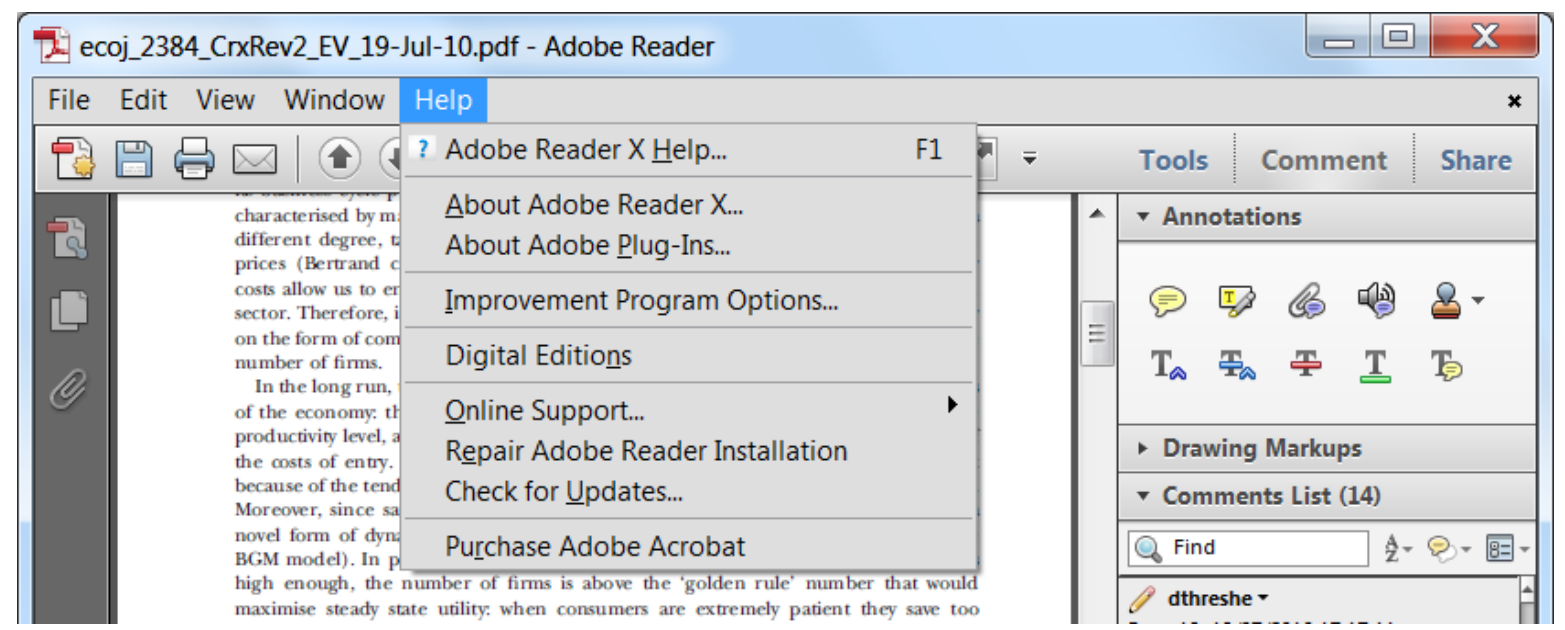

